\title{
Early noninvasive ventilation failure in COPD with acute on chronic respiratory failure
}

\section{To the Editors:}

Noninvasive ventilation (NIV) has proven to be of great interest in the management of acute respiratory failure (ARF) due to exacerbations of chronic obstructive pulmonary disease (COPD), with an improvement in arterial blood gas tensions and respiratory frequency usually observed $1-6 \mathrm{~h}$ after initiation [1, 2]. We describe an unusual and reversible condition of early NIV failure as follows.

The patient was a 72-yr-old male with chronic respiratory failure from COPD, successfully treated with nocturnal bilevel pressure NIV for 20 months. He was hospitalised in our respiratory intensive care unit (CHU de Rouen, Hôpital de Bois-Guillaume, Rouen, France) for ARF ( $\mathrm{pH}=7.26$; arterial carbon dioxide tension $\left(\mathrm{Pa}, \mathrm{CO}_{2}\right) 11.4 \mathrm{kPa}$; arterial oxygen tension $\left(\mathrm{Pa}_{\mathrm{O}} \mathrm{O}_{2}\right)$ / inspiratory oxygen fraction $\left.\left(\mathrm{FI}, \mathrm{O}_{2}\right) 338\right)$. The patient presented with bilateral wheezing, encephalopathy and gaseous abdominal distension. Standard medical treatment associated with NIV failed to improve the patient's condition, despite different settings with pressure-preset and flow-preset ventilatory modes, and careful management with facial and nasal masks. Five hours after admission, the patient's condition worsened $(\mathrm{pH}=7.12$; $\left.\mathrm{Pa}, \mathrm{CO}_{2} 19.2 \mathrm{kPa} ; \mathrm{Pa}_{2} \mathrm{O}_{2} / \mathrm{FI}_{1} \mathrm{O}_{2} 115\right)$. Intubation was then performed, subsequently revealing a laryngeal vestibule carcinoma, partially obstructing the glottis aperture. After tumoural laser resection, the patient was successfully weaned, extubated and then discharged home with his previous NIV treatment.

Early NIV failure in COPD with ARF may be ascribed to the patient's clinical status, such as bronchial hypersecretion, deterioration in medical condition, pneumothorax, haemodynamic instability, severe encephalopathy or gastric distension. Technical ventilator-associated factors could also account for early NIV failure, such as inadequate settings and/or ventilatory mode, inadequate inspiratory and/or expiratory triggering, deleterious leaks, excessive dead space and rebreathing. Preliminary data suggest that patient-ventilator asynchronism and NIV failure could also result from underestimated high nasal and/or upper airway resistances, particularly reflex glottic narrowing [3]. In this respect, upper airway tumours may cause progressive airway obstruction, can mimic a COPD exacerbation and lead to difficulties during NIV, except if their presence is clinically suspected by a stridor. Indeed, NIV is contraindicated in this latter situation, and the patients should be intubated. In patients with ARF treated by invasive mechanical ventilation, local tumoural resection by bronchoscopic intervention has been shown to be associated with successful weaning in the majority of cases and to be a cost-saving approach [4].

During chronic obstructive pulmonary disease exacerbation requiring noninvasive ventilation, we suggest that an unexplained early noninvasive ventilation failure should lead to an evaluation of the integrity of the extra- and intrathoracic upper airways before or during the mandatory intubation procedure. Fibreoptic bronchoscopy via a facial mask may facilitate an early diagnosis, but still needs to be evaluated for patients with this condition [5].

\section{A. Cuvelier, L.C. Molano and J-F. Muir}

Respiratory and Intensive Care Medicine, CHU de Rouen, Hôpital de Bois-Guillaume, Rouen, France.

\section{REFERENCES}

1 Brochard L, Mancebo J, Wysocki M, et al. Noninvasive ventilation for acute exacerbations of chronic obstructive pulmonary disease. N Engl J Med 1995; 333: 817-822.

2 Mehta S, Hill NS. Noninvasive ventilation. Am J Respir Crit Care Med 2001; 163: 540-577.

3 Jounieaux V, Aubert G, Dury M, Delguste P, Rodenstein DO. Effects of nasal positive-pressure hyperventilation on the glottis in normal awake subjects. J Appl Physiol 1995; 79: 176-185.

4 Colt HG, Harrell JH. Therapeutic rigid bronchoscopy allows level of care changes in patients with acute respiratory failure from central airways obstruction. Chest 1997; 112: 202-206.

5 Antonelli M, Conti G, Riccioni L, Meduri GU. Noninvasive positive-pressure ventilation via face mask during bronchoscopy with BAL in high-risk hypoxemic patients. Chest 1996; 110: 724-728.

\section{Antibody deficiency in bronchiectasis}

\section{To the Editors:}

We have read with interest the article by VAN KESSEL et al. [1] on impaired antibody response to the pneumococcal polysaccharides of Streptococcus pneumoniae in patients with bronchiectasis of unknown aetiology. Several aspects of this work are controversial and there is no unanimous agreement on some of their conclusions. The first aspect that merits attention is the lack of a control group to establish the normal total antibody response and the isotypes to the vaccine, and, consequently, 
the diagnostic criteria of impaired antibody response. There are no universal criteria for adequate antibody response to polysaccharides and each laboratory should establish its own [2]. This is particularly important because of the therapeutic implications. Treatment of symptomatic patients with a lack of antibody response is performed with periodic administration of intravenous immunoglobulins (Ig) at a high cost, dependent on healthcare centres and potential side-effects [3]. For this reason, strict diagnostic criteria must be established.

It is interesting to note that VAN KESSEL et al. [1] used different criteria for the response with total antibodies to the serotypes 3,4 and $9 \mathrm{~V}$, in which the post-vaccination titre should be $>20 \mathrm{U} \cdot \mathrm{mL}^{-1}$ with at least a two-fold increase for two of the three serotypes, and to the isotypes $\operatorname{IgA}$ and $\operatorname{IgG} 2$ of the six serotypes studied, in which response was defined individually for each serotype as post-immunisation concentrations $>50$ $\mathrm{U} \cdot \mathrm{mL}^{-1}$ and an isotype responder had to have a positive $\operatorname{IgA}$ and/or IgG2 response to more than four out of six of the serotypes. The reason for these criteria are not clear and should be based on the knowledge of the response of healthy individuals. With their criteria, a patient could potentially be classified as a reponder if he/she presented post-immunisation concentrations of $\operatorname{IgA}>50 \mathrm{U} \cdot \mathrm{mL}^{-1}$ to five of the six serotypes, but with low concentrations of IgG2 to all serotypes. The clinical meaning of the antibody response with the different isotypes is not equivalent. As an example, the clinical manifestations of patients with IgA deficiency in the form of respiratory infections are due, in great part, to the possible association of an IgG-subclass deficiency, particularly IgG2 deficiency $[4,5]$.

The usefulness of their response criteria can be validated, at least in part, with the clinical characteristics of the responder and nonresponder patients. There were very few, nonsignificant and probably not clinically meaningful differences between the group of responders and nonresponders. Only the number of resected segments was significantly different in both groups, but it would be interesting to see the number of patients requiring surgery in both groups. This result could be subjected to possible bias in a case in which a single severe patient requiring extensive surgery was included in the group of nonresponders [1].

The next query refers to the meaning of the lack of response to individual serotypes of $S$. pneumoniae. The immunological interest is clear, but the clinical meaning is controversial. It would be very informative to know the clinical characteristics of the four patients classified as nonresponders by showing post-vaccination total antibody titres below the threshold and comparing them with the remaining 22 patients [1]. In contrast, the lack of response with a particular isotype of Ig to one or some individual serotypes can potentially be less harmful than the lack of response with total IgG or IgG2 to all serotypes considered together. This last condition may be a marker of a more profound impairment in antibody response and, if this impairment is also observed against conjugated polysaccharides such as the Haemophilus influenzae type B (Hib) vaccine in a patient with recurrent respiratory infections, it is a criterion for Ig replacement therapy [6]. This lack of response to both vaccines is a characteristic of patients with a severe immune impairment [6]; however, it is not at all clear that the lack of response restricted to one or more unconjugated polysaccharides deserves treatment with Ig. VAN KESSEL et al. [1] mention that without the study of the response to different pneumococcal serotypes with both isotypes, IgA and/or IgG2, 11 out of 15 so-called nonresponders would have been missed, but there is no convincing clinical or immunological evidence that these individuals had a worse prognosis and were, therefore, candidates for Ig replacement therapy.

By studying a group of healthy adults and a group of patients with humoral immunodeficiencies characterised by defective antibody formation, RODRIGO and coworkers $[6,7]$ were able to establish a response criteria to both the pneumoccocal and the conjugated Hib vaccines. It was observed that not all healthy individuals responded adequately to either vaccine with all isotypes, but, conversely, no healthy subject presented a lack of response to both vaccines [6]. In contrast, none of the patients with humoral immunodeficiencies responded to either vaccine. Therefore, it was shown that evaluation of the antibody response to both the conjugated and nonconjugated vaccine allows the diagnosis of the humoral immunodeficiency characterised by a lack of antibody response to polysaccharides to be established, and permits the selection of patients as candidates to receive Ig therapy.

By using these criteria based on lack of response to both vaccines, we could observe an antibody-production deficiency with normal IgG levels in $11 \%$ of a group of 107 patients with bronchiectasis of unknown aetiology. The nonresponders had a significantly higher incidence of otitis media, lower serum IgG2-subclass levels and lower pre-immunisation antibody titres to S. pneumoniae and Hib, and had recurrent pneumonia more frequently (in this latter case, differences were not significant due to the low number of nonresponders) [8], similar to the first case described by AMBROsino et al. [9].

We totally agree that a sizeable fraction of patients with bronchiectasis of unknown cause can now be classified as bronchiectasis associated with polysaccharide antibodyresponse deficiency $[1,8,10,11]$. However, before this diagnosis can be established and substitution therapy with immunoglobulins can be indicated, comprehensive immunological evaluation is mandatory. This evaluation should include analysis of antibody response to a conjugated and unconjugated vaccine, and response criteria must be defined based on the response of a healthy adult population [6, 7].

\section{Miravitlles*, M. Vendrell ${ }^{\#}$ and J. de Gracia}

*Servei de Pneumologia, Institut Clínic del Tòrax (IDIBAPS), Hospital Clínic, and Servei de Pneumologia, Hospital Universitari Vall d'Hebron, Barcelona, and "Secció de Pneumologia, Hospital Josep Trueta, Girona, Spain.

\section{REFERENCES}

1 van Kessel DA, van Velzen-Blad H, van den Bosch JMM, Rijkers GT. Impaired pneumococcal antibody response in bronchiectasis of unknown aetiology. Eur Respir J 2005; 25: 482-489.

2 Go ES, Ballas ZK. Anti-pneumococcal antibody response in normal subjects: a meta-analysis. J Allergy Clin Immunol 1996; 98: 205-215. 
3 de Gracia J, Vendrell M, Álvarez A, et al. Immunoglobulin therapy to control lung damage in patients with common variable immunodeficiency. Int Immunopharmacol 2004; 6: 745-753.

4 de Gracia J, Morell F, Bofill JM, Rodrigo MJ, Cosculluela C. Impaired lung function in patients with $\operatorname{IgA}$ deficiency and low levels of IgG2 or IgG3. N Engl J Med 1986; 314: 925-926.

5 de Gracia J, Miravitlles $M$, Vendrell $M$, Rodrigo MJ, Codina R, Morell F. Study of the IgG subclasses in symptomatic patients with IgA deficiency. Med Clin (Barc) 1995; 104: 728-731.

6 Rodrigo MJ, Vendrell M, Cruz MJ, et al. Utility of the antibody response to a conjugated $H$. influenzae type $\mathrm{B}$ vaccine for diagnosis of primary humoral immunodeficiency. Am J Respir Crit Care Med 2000; 162: 1462-1465.

7 Rodrigo MJ, Miravitlles M, Cruz MJ, et al. Characterization of specific immunoglobulin G (IgG) and its subclasses (IgG1 and IgG2) against the 23-valent pneumococcal vaccine in a healthy adult population: proposal for response criteria. Clin Diagn Lab Immunol 1997; 4: 168-172.

8 Vendrell M, de Gracia J, Rodrigo MJ, et al. Antibody production deficiency with normal IgG levels in bronchiectasis of unknown etiology. Chest 2005; 127: 197-204.

9 Ambrosino DM, Siber GR, Chilmonczyk BA, Jernberg JB, Finberg RW. An immunodeficiency characterized by impaired antibody responses to polysaccharides. $N$ Engl J Med 1987; 316: 790-793.

10 de Gracia J, Rodrigo MJ, Morell F, et al. IgG subclass deficiencies associated with bronchiectasis. Am J Respir Crit Care Med 1996; 153: 650-655.

11 Miravitlles M, de Gracia J, Rodrigo MJ, et al. Specific antibody response against the 23-valent pneumococcal vaccine in patients with $\alpha_{1}$-antitrypsin deficiency with and without bronchiectasis. Chest 1999; 116: 946-952.

\section{Combination therapy with bosentan and phosphodiesterase-5 inhibitor in pulmonary arterial hypertension}

\section{To the Editors:}

HOEPER et al. [1] have produced an interesting report of their clinical experience of combined therapy with bosentan and sildenafil in patients with idiopathic pulmonary arterial hypertension. Whilst it is true that the relatively scant literature supporting the use of phosphodiesterase- 5 inhibitors is centred around sildenafil, its relatively short duration of action requires the use of a thrice-daily regime. This has significant implications for compliance and, since treatment is continual, has large implications in the cost of treatment. It would be more logical to use a long-acting phosphodiesterase-5 inhibitor, and, with the advent of tadalafil, once-daily treatment becomes possible.

Here, we report our experience with a combination of bosentan and tadalafil in a 42-yr-old male with idiopathic pulmonary hypertension who had documented poor compliance with nebulised iloprost. Sildenafil $25 \mathrm{mg}$ t.d.s. was added to bosentan, following a clinical deterioration and the finding of an estimated pulmonary artery systolic pressure of $130 \mathrm{mmHg}$, and this caused a fall in pulmonary artery pressure to $50 \mathrm{mmHg}$. Treatment with sildenafil was stopped 1 month later at another centre, and the pulmonary artery systolic pressure increased to $100 \mathrm{mmHg}$. Subsequently, sildenafil was restarted in combination with bosentan, and exercise tolerance doubled. After 3 months of combination treatment, the issue of compliance was raised by the patient and it was decided to substitute tadalafil $20 \mathrm{mg}$ once daily for the sildenafil. The patient has continued on tadalafil for 9 months with an excellent symptomatic response. The last estimated pulmonary artery pressure was $61 \mathrm{mmHg}$.

\section{A.H. Morice, S. Mulrennan and A. Clark}

Division of Academic Medicine, University of Hull, Castle Hill Hospital, Hull, UK.

\section{REFERENCE}

1 Hoeper MM, Faulenbach C, Golpon H, Winkler J, Welte T, Niedermeyer J. Combination therapy with bosentan and sildenafil in idiopathic pulmonary arterial hypertension. Eur Respir J 2004; 24: 1007-1010.

DOI: 10.1183/09031936.05.00030405

\section{From the authors:}

I am grateful for the comments by A.H. Morice and coworkers, although their case report leaves several questions unanswered. More information than just the systolic pulmonary artery pressure is needed to appraise the haemodynamic response to tadalafil. One wonders whether this patient ever underwent pulmonary vasoreactivity testing. With such a 